\title{
Alimentação e sustentabilidade
}

\author{
HELENA RIBEIRO ${ }^{I}$, \\ PATRÍCIA CONSTANTE JAIME ${ }^{I}$ \\ e DEISY VENTURA ${ }^{I}$
}

\section{Introdução}

A

ALIMENTAÇÃo é uma atividade que envolve muito mais que o ato de comer e a disponibilidade de alimentos. Há uma cadeia de produção, que se inicia no campo, ou antes, na preparação de sementes, mudas e insumos, passando por ciclos, do plantio à colheita, em que elementos da natureza têm um papel crucial, mas que vêm sendo, cada vez mais, envolvidos por questões tecnológicas, financeiras e sociais. Nas etapas produtivas, no campo, as inter-relações com a sustentabilidade parecem claras. De fato, o próprio termo sustentabilidade foi cunhado com forte influência da atividade agrária. No entanto, as etapas posteriores, até que o alimento chegue a nossas mesas e, posteriormente, seu descarte envolvem questões complexas, que não são abarcadas por uma única área de conhecimento, e que possuem uma dinâmica cotidiana crescentemente artificializada e acelerada. Em todo esse amplo, diverso e complexo trajeto do solo ao prato há inúmeras interfaces com a in/sustentabilidade que precisam ser continuamente apreendidas e entendidas. Assim, o tema da alimentação e sua relação com a sustentabilidade planetária é muito antigo e, ao mesmo tempo, muito atual, portanto de fronteira, no sentido temporal, de vanguarda. Ao exigir olhares, investigações e soluções multidisciplinares é, também, um tema de fronteira de conhecimentos, por conta de suas múltiplas interfaces.

Este ensaio, de autoria interdisciplinar, tem como objetivo pincelar um panorama e apresentar uma reflexão sobre as complexas e intrincadas questões que envolvem a alimentação, sob uma perspectiva da sustentabilidade global.

\section{Produção de alimentos}

A discussão sobre alimentação e sustentabilidade se inicia com a questão se será possível a terra alimentar nove bilhões de habitantes, previstos para viver no planeta em 2050 (Conte; Boff, 2013) sem degradá-la de modo irreversível e com dieta alimentar que contribua para a sustentabilidade, ao mesmo tempo que garanta a saúde e o bem-estar das pessoas. Morin (2013, p.269) destaca que o "problema da agricultura é de âmbito planetário, indissociável do problema da água, da demografia, da urbanização, de ecologia (mudanças climáticas), bem como, sem dúvida, o da alimentação, eles mesmos problemas interdependentes uns dos outros".

No entanto, segundo Cassol e Schneider (2015), 
No âmbito de estudos rurais, as discussões em torno da alimentação ganharam impulso a partir da consolidação do processo de globalização da produção e distribuição de alimentos, que passou a se concentrar cada vez mais nas mãos das grandes empresas transnacionais Mais recentemente, outros elementos também passaram a impulsionar a problemática sociológica dos alimentos, como (1) as questões de saúde pública (desnutrição e obesidade), (2) os problemas ambientais decorrentes da produção de alimentos (poluição e contaminação com agroquímicos) e (3) a opulência do consumo e o consequente desperdício de alimentos.

Uma crise agrícola, que atinge camponeses desenraizados e os habitantes urbanos do Sul, segundo Morin (2013), provém dos efeitos da mundialização de tripla face (globalização, desenvolvimento, ocidentalização), da expansão descontrolada da economia capitalista, com a extensão da agricultura especializada e todas as suas consequências.

Estudos recentes mostram que a questão alimentar extrapola a dimensão da oferta de alimentos e os processos de organização produtiva. Carolan (2012 apud Cassol; Schneider, 2015 ) considera que o estudo das relações de consumo e dos sistemas de produção agroalimentares é fundamental para a compreensão do comportamento e das ações dos indivíduos na sociedade moderna, assim como a conexão com a saúde coletiva. O ato de comer é uma ação social com sentido capaz de gerar novos valores e modos de vida sustentáveis.

Ainda segundo Cassol e Schneider (2015), a interação entre as formas de produzir e comercializar e os modos de consumir e alimentar são cruciais para desenvolver práticas sustentáveis, tanto de produção quanto de consumo.

No entanto, a preocupação com formas mais sustentáveis e saudáveis de se produzir alimentos não é tão recente e tem motivado alertas da comunidade científica há algumas décadas. Mencionamos, aqui, duas obras-chave nesse contexto.

A primeira delas é o famoso best-seller mundial de Rachel Carson, Silent Spring, ou "Primavera silenciosa" em português (Carson, 1962), que, há mais de 50 anos, alertava para os riscos do uso massivo de pesticidas:

Eu não defendo que inseticidas químicos não devem ser usados nunca, eu argumento que colocamos indiscriminadamente produtos químicos venenosos e biologicamente potentes na mão de pessoas muito ou totalmente ignorantes de seus danos potenciais. Nós submetemos números enormes de pessoas ao contato com estes venenos sem o seu consentimento e, frequentemente, sem seu conhecimento. (Carson, 1962, p. 22 - Tradução livre das autoras)

Numa era de especialistas, cada um vê seu problema e não tem consciência ou é intolerante com um quadro mais amplo em que ele se insere. Também é uma era dominada pela indústria, na qual o direito a ganhar um dólar a qualquer custo raramente é desafiado. (ibidem, p.23) 
Os pesticidas foram desenvolvidos e usados nos dois últimos séculos, mas de forma mais intensa a partir da Segunda Guerra Mundial, para combater as pragas em lavouras e controlar vetores de doenças. Uma praga é qualquer espécie de planta, animal, ou micro-organismo que ameace a saúde e o bem-estar humanos. No entanto, a maioria delas se encaixa em nichos ecológicos específicos e tem funções importantes para a integridade dos ecossistemas, inclusive quando não são diretamente úteis aos humanos. Assim, uma praga raramente é só uma praga (Robson; Hamilton, 2010). Atualmente, o uso maciço de fertilizantes químicos e agrotóxicos tem levado à poluição de cursos d'água, lençóis freáticos e solos, em todo o mundo, com consequências ecológicas e sanitárias muito nefastas, além do empobrecimento da biodiversidade (Morin, 2013). A saúde pública e a pesquisa ecológica têm revelado, cada vez mais, problemas com o uso de pesticidas, que são tóxicos para os seres humanos e animais silvestres e perturbadores dos ecossistemas naturais (Robson; Hamilton, 2010). Já em 2010 existiam 900 ingredientes ativos de uso comercial, usados em formulação de 35 mil produtos comercializados para controle de pragas. Esses produtos são tóxicos de forma aguda, podendo levar a dores de cabeça, hipersecreção, movimento muscular, náusea, diarreia e até à morte, em casos mais severos. Adicionalmente, a intoxicação crônica é uma preocupação crescente, pelo acúmulo de evidências de que estaria associada a diferentes tipos de câncer e a efeitos crônicos no sistema nervoso central e enfermidades crônicas neurodegenerativas, incluindo doença de Parkinson (Robson; Hamilton, 2010).

Essas preocupações e evidências têm levado à elaboração de regulamentações para uso desses produtos, em todo o mundo. Além disso, têm levado ao desenvolvimento de novas formas de combate a pragas, em que se destaca o Manejo Integrado de Pragas, um enfoque que utiliza diversas técnicas de controle (físico, mecânico, biológico e químico, além de monitoramento de pragas, educação dos consumidores, técnicas culturais de manejo, salubridade e manejo de resíduos sólidos) para manter, ou administrar, a população de pragas em níveis inferiores aos que provocam dano econômico, ao mesmo tempo que garantam a qualidade do ambiente e protejam a saúde humana (Robson; Hamilton, 2010). A agricultura orgânica é uma outra opção, que tem recebido, cada vez mais, adesão de consumidores, juntamente com os alimentos naturais, que são aqueles que recebem um processamento mínimo e não possuem ingredientes artificiais (McSwane, 2010).

Outra obra pioneira na introdução de uma abordagem mais integrada à alimentação humana foi Diet for a Small Planet, que trouxe e discutiu o impacto da dieta humana em questões relacionadas à segurança alimentar e à sustentabilidade do nosso planeta (Lappé, 1976). Na década de 1970, já alertava que o discurso de limite da capacidade alimentícia da terra estava sendo usado para beneficiar sistemas produtivos de alimentos e que havia em curso um processo para reduzir essa capacidade alimentícia, via incentivo à produção e ao consumo 
de carne e de alimentos ultraprocessados. Assim, a capacidade de resolver a fome e a carência de alimentos no mundo era um problema profundamente relacionado a questões políticas e econômicas. Na mesma época, apareceu, também, a questão: como nossas dietas se relacionam a aspectos mais amplos de provimento de alimentos para toda humanidade? Era preciso entender como fatores econômicos e não naturais ou agrícolas determinavam o uso da terra e de alimentos (Lappé, 1976). Ao se entender o mundo através da alimentação ficava claro que qualquer sistema econômico deveria ser julgado, sobretudo, pela forma como produz e usa seus recursos alimentares. Em escala global, o sistema produtivo causa destruição e desperdício de alimentos de forma invisível e praticamente desconhecida do grande público. Enquanto os pobres não podem pagar, os agricultores e as indústrias alimentícias vendem e adquirem alimentos em grãos e os processam de forma a que se transformem em ração para gado, ou alimentos pouco nutritivos. Mudando a base de nossa alimentação para proteínas de origem vegetal, se poderia maximizar o potencial do planeta de suprir as necessidades nutricionais, com alimentos mais saudáveis e agradáveis, e, ao mesmo tempo, minimizar a destruição de ecossistemas naturais para transformá-los em agropecuários (Lappé, 1976).

A base do atual sistema produtivo foi a Revolução Verde, a partir da década de 1950, que permitiu um aumento incrível da produtividade das lavouras norte-americanas, via monoculturas especializadas, com melhoramento genético de sementes, e uso disseminado de fertilizantes químicos e pesticidas, cujos problemas já haviam sido alertados por Carson (1962). Na década de 1970, a Revolução Verde foi exportada para a Ásia e, finalmente, disseminada para todo o mundo, aumentando enormemente a disponibilidade de alimentos, e baixando seu custo. Para dar destino a essa superprodução é que se passou a propagandear um maior consumo de carne, sobretudo bovina, além das necessidades proteicas humanas. Um bovino consegue reduzir 7 a 16 quilos de grão ou de soja, por ele ingerido, a l quilo de carne (Lappé, 1976). O restante fica inacessível para consumo humano. Essa equação levou a um encarecimento do preço de alimentos, que se tornaram mais rentáveis, prejudicando as camadas mais pobres da população. De 1970 a 2010, o consumo médio mundial anual de carne passou de 25 para 38 quilos por pessoa (Morin, 2013).

O outro lado da moeda foi fazer alimentos ultraprocessados mais baratos, mas de muito baixo poder nutritivo, que passaram a ser consumidos largamente por aqueles de renda mais baixa (Monteiro; Cannon, 2012).

Assim, há uma grande transição nutricional em curso, com adoção de dietas ocidentalizadas, baseada em elevado consumo de carne e produtos lácteos, ao lado de alimentos ultraprocessados, por quase todos os povos, que tem levado a um aumento acelerado da obesidade e da incidência de doenças crônicas.

Além disso, há uma situação global paradoxal, que leva alguns países a favorecem as exportações agrícolas, em detrimento de sua soberania alimentar, 
que permitiria alimentar sua população de maneira autônoma, principalmente em cereais. Um exemplo é o da África, grande exportadora de frutas e produtos tropicais, mas que precisa importar um terço de suas necessidades (Morin, 2013). Concomitante, há ainda aproximadamente 800 milhões de pessoas que passam fome e dois milhões de pessoas com deficiência de micronutrientes, no mundo (The Lancet, 2016).

Percebe-se, assim, que o modelo agrícola de desenvolvimento, adotado a partir da Revolução Verde, se, por um lado, promoveu aumento na produtividade, expansão das fronteiras agrícolas e diminuição da penosidade do trabalho, por meio da intensificação do uso de máquinas agrícolas; por outro, resultou em estimulo à utilização de sementes híbridas, fertilizantes químicos, agrotóxicos e drogas veterinárias, uso intensivo do solo, redução da biodiversidade, êxodo rural e aumento da concentração fundiária. Não obstante, a realidade de fome e insegurança alimentar não foi reduzida. Contrariamente, o que se observou foram efeitos negativos à saúde humana (Kathounian, 2001; Navolar et al., 2010).

Nesse sentido, Maluf et al. (2015) discutem o papel dos modelos agrícolas na promoção da saúde e apresentam a importância socioeconômica e ambiental da agricultura familiar e seu potencial para contribuir para o que denominam "Agricultura Sensível à Nutrição". Esse conceito orienta-se pela perspectiva dos Determinantes Sociais da Saúde e considera que a promoção da alimentação saudável, e com ela a garantia de segurança alimentar e nutricional, derivará de sistemas alimentares mais justos socialmente e ambientalmente sustentáveis. Uma Agricultura Sensível à Nutrição seria, portanto, orientada pelo modelo da agricultura familiar de base agroecológica, para obtenção de maior autonomia dos agricultores perante as grandes corporações de produção de alimentos e garantia de sistemas de produção baseados em circuitos que aproximam quem produz e quem consome o alimento. Portanto, a importância da diversidade de alimentos produzidos e consumidos ganha reconhecimento, unindo as pontas entre produção e consumo de uma dieta saudável (FAO, 2010).

\section{O ambiente alimentar}

Em termos de saúde pública, após um período de maior disponibilidade de alimentos e uma consequente queda na prevalência de fome, em percentagem da população mundial, as doenças crônicas estão emergindo como novas epidemias urbanas. Apesar de não exclusivas das cidades, o ambiente urbano propicia estilos de vida e comportamentos que contribuem ao seu desenvolvimento (WHO; UN-Habitat, 2016). Como exemplo, o documento cita o mau planejamento urbano, as longas horas no transporte, a elevada dependência no transporte motorizado que podem levar à falta de atividade física e à dificuldade de se fazer escolhas alimentares mais sadias. As doenças crônicas eram consideradas doenças de afluência, mas, hoje em dia, matam mais pessoas nos países em desenvolvimento que naqueles mais desenvolvidos, apesar de neles ainda continuarem como problemas importantes. 
O ambiente urbano facilita muito causas primárias e fatores de risco para doenças crônicas: o ambiente alimentar urbano, o desenho espacial das cidades, os sistemas de transporte, condições de habitação e de vizinhança, e violência contribuem para a carga de doenças crônicas, ao lado de outros determinantes importantes, como educação, emprego e renda. Por outro lado, governos locais que têm a autoridade para atuar na melhoria do ambiente urbano - em habitações, calçadas, parques, ruas, transporte público, o têm feito de modo restrito.

A alimentação e a nutrição influenciam a saúde e o bem-estar das pessoas, assim como outros determinantes de saúde, tais como educação e emprego, durante toda a vida.

Estudos da Organização Mundial de Saúde (OMS) têm mostrado taxas crescentes de obesidade em países de renda baixa e média, com destaque para a América Latina e o Caribe. Pesquisas têm mostrado, também, que a obesidade em populações urbanas é mais elevada entre os mais pobres e os com menor nível educacional (WHO; UN-Habitat, 2016). Entretanto, os níveis de obesidade coexistem com subnutrição em algumas regiões mais pobres.

O documento da OMS e UN-Habitat (2016) ressalta que as ações em questões nutricionais não podem se limitar ao setor saúde, apesar de esse ter um papel relevante. Destaca que serviços urbanos básicos, como abastecimento de água, saneamento e higiene têm impacto grande nas condições nutricionais, ao reduzir episódios de diarreia. $\mathrm{O}$ desenho urbano e as estruturas que favorecem a atividade física e o acesso a alimentos saudáveis e a agricultura urbana, também, são considerados, hoje em dia, como pontos importantes de políticas de alimentação, visando garantir a segurança alimentar. Usos mistos do solo e maior densidade urbana estão correlacionados com níveis mais altos de atividade física e menor prevalência de obesidade.

\section{Novo paradigma sobre Alimentação Saudável}

Por muito tempo, o conceito de alimentação saudável não adotou uma visão sistêmica da alimentação, que considera o conjunto de etapas pelas quais os alimentos passam, desde sua produção, extração, processamento, distribuição e comercialização, até o consumo humano, no final desta cadeia de processos sociais.

A abordagem conceitual, que reduz a alimentação saudável à composição química dos alimentos e ao papel que determinados nutrientes, como gordura, sódio e fibras, exercem sobre a saúde foi denominada nutricionismo por Michael Pollan (2008), em seu livro Em defesa da comida: um manifesto.

Profundas mudanças nos padrões de alimentação da população mundial são observadas, a partir da segunda metade do século passado, com enfraquecimento dos padrões alimentares tradicionais e substituição pelo consumo de alimentos processados industrialmente, com alta densidade energética, altos teores de açúcares, sódio, gorduras saturadas e trans, e baixo conteúdo de fibras (Popkin, 2006; 2014). Nutrientes isoladamente passam a explicar pouco a relação entre alimentação e saúde. 
Dessa forma, no campo da saúde coletiva emerge o debate sobre o impacto dos modelos hegemônicos de produção e processamento de alimentos sobre os padrões alimentares populacionais. Oficialmente, a Organização Mundial de Saúde reconhece o impacto do processamento industrial de alimentos na saúde da população, em 2003 (WHO, 2003).

Monteiro et al. (2015) apresentam um novo paradigma sobre alimentação saudável, orientado pela classificação de alimentos que considera a extensão e o propósito do processamento industrial a que foram submetidos os alimentos antes de sua aquisição e consumo pelos indivíduos. Essa classificação aloca os itens alimentares em quatro grandes grupos. O primeiro é composto pelos alimentos in natura, ou minimamente processados, do qual fazem parte alimentos extraídos da natureza para serem consumidos logo após sua coleta, ou que passaram por processamento mínimo, com a finalidade de aumentar sua duração e, às vezes, facilitar o seu preparo. Ao segundo grupo pertencem os ingredientes culinários processados, substâncias extraídas de alimentos ou da natureza e utilizados nas preparações culinárias, como óleo, açúcar e sal. O terceiro grupo, de alimentos processados, abrange produtos manufaturados essencialmente com a adição de sal, açúcar ou óleo a alimentos in natura ou minimamente processados, como conservas de legumes, carnes salgadas, queijos e pães do tipo artesanal. O quarto grupo, por sua vez, difere-se dos demais por ser composto por novas criações industriais, contendo pouco ou nenhum alimento inteiro, ao qual são empregadas modernas e sofisticadas tecnologias, como a intensiva utilização de aditivos alimentares, que conferem aos produtos cor, sabor, aroma e textura hiperatrativos (Monteiro et al., 2016).

Em países desenvolvidos, como Reino Unido e Canadá, a participação de alimentos ultraprocessados, no total de calorias consumidas, é de 63,4\% (Moubarac et al., 2013) e 55\% (Moubarac et al. 2014), respectivamente. Entre os países da América Latina, Chile e México destacam-se pelo maior consumo de alimentos ultraprocessados, respectivamente $55 \%$ (Crovetto et al., 2014) e 58\% das calorias disponíveis para consumo (Popkin, 2014). No Brasil, a contribuição calórica para consumo desses produtos é igual a 25,4\% (Martins et al., 2013).

Há uma intrínseca relação entre a produção de alimentos ultraprocessados e o modelo do agronegócio que, atualmente, se volta para os mercados internacionais de commodities agropecuárias, tais como grãos e carnes (Schneider, 2010). Esses mercados caracterizam-se, entre outros aspectos, pela produção de insumos para a indústria dos produtos alimentares ultraprocessados (Monteiro et al., 2013). Além do frequente e abusivo uso de agrotóxicos, do consumo de água e emissão de dióxido de carbono e metano, dentre outros gases de efeito estufa, na produção, transporte e processamento de alimentos, outra preocupação ecológica decorrente da tendência de aumento de consumo de alimentos ultraprocessados é a gestão insuficiente de seus resíduos ambientais, em especial das embalagens. 
A geração mundial de Resíduos Sólidos Urbanos (RSU) é de cerca de 1,3 bilhão de toneladas/ano e estima-se que haverá um aumento para aproximadamente 2,2 bilhões de toneladas/ano, até 2025. Isso representa um aumento da taxa média de geração per capita de 1,2 para $1,42 \mathrm{~kg} / \mathrm{hab}$./dia (World Bank, 2010). A geração per capita de resíduos está aumentando em quase todo o mundo. Desse total, aproximadamente $32 \%$ são resíduos secos, principalmente embalagens. A má gestão dos resíduos sólidos urbanos, sobretudo em regiões mais pobres carentes de infraestrutura de saneamento, tem propiciado formação de focos de reprodução mosquitos transmissores de doenças infecciosas, tais como dengue, chikungunya e o vírus zika.

Percebe-se, assim, que uma visão ampliada de alimentação saudável e sustentável envolve saberes e práticas de diversos atores e campos do conhecimento que se relacionam com a alimentação e o sistema alimentar. Dessa forma, torna-se importante compreender não só o que se come, mas também as formas de produção (modelos de agricultura e pecuária) e de acesso aos alimentos (políticas de abastecimento e comercialização, em diferentes modalidades) e os aspectos da comensalidade, ou seja, os modos de comer de indivíduos e coletividades (Monteiro et al., 2015). Com sistemas alimentares cada vez mais globalizados, os mecanismos de regulação de alimentos assumem condição sine qua non para agenda global de segurança alimentar e nutricional.

\section{A regulação internacional da produção de alimentos: o caso do Codex Alimentarius}

Os mercados internacionais de alimentos caracterizam-se por um quadro regulatório complexo. A partir dos anos 1990, os padrões técnicos e normas relacionadas a qualidade, inocuidade e outros atributos de produtos e de processos produtivos passaram a ser elementos estruturais desses mercados, capazes de afetar seriamente a competitividade de cadeias produtivas e causar impactos econômicos e sociais negativos e diferenciados em países e/ou em regiões (Martinelli Jr., 2013, p.522).

O Codex Alimentarius (do latim Lei ou Código dos Alimentos) é incontestavelmente o corpus juris mais importante no âmbito do comércio mundial de produtos alimentares (Mbengue; Thomas, 2004, p.230). Trata-se de uma coletânea de normas alimentares adotadas no plano internacional, apresentadas de modo uniforme, compreendendo diretrizes, códigos de práticas e recomendações. Ele é elaborado por uma Comissão que foi instituída em 1961 pela OMS e pela FAO, denominada Comissão do Codex Alimentarius (CCA), que reúne, anualmente, seus 188 membros (187 Estados e a União Europeia) e 234 observadores, dos quais 54 são organizações intergovernamentais, 164 são Organizações Não Governamentais e 16 são organismos das Nações Unidas (OMS; FAO, 2016).

A importância do Codex Alimentarius cresce, sobretudo, após a criação da Organização Mundial de Comércio (OMC), pois o sistema de solução de con- 
trovérsias da organização reconhece as normas do Codex, em particular quando se discute a aplicação dos seus acordos sobre Medidas Sanitárias e Fitossanitárias (AMSF) e sobre Barreiras Técnicas ao Comércio (ABTC). Logo, da condição de meros padrões ou recomendações, o cumprimento das normas do Codex, pelos Estados-membro da OMC, passou a ser exigido por intermédio das decisões tomadas no âmbito de seu sistema de solução de controvérsias (Lassalle-de Sallins, 2009 , p.146). Embora os acordos da OMC não tenham tornado as normas do Codex obrigatórias, graças à interpretação que os grupos especiais e o Órgão Permanente de Apelação da OMC fazem delas, a eventual inconformidade de uma medida nacional com o Codex é, de fato, difícil de justificar perante a OMC (Culot, 2005, p.283).

Tal convergência explica-se, em grande parte, porque tanto o AMSF da OMC como o Codex Alimentarius "tratam os produtos principalmente como commodities e não como alimentos em sua dimensão total e em sua importância vital para os seres humanos", seguindo "as diretrizes e a lógica do sistema industrial de produção, que direciona hoje toda a produção agropecuária", em detrimento da necessária resposta internacional aos "graves problemas do acesso da população à comida (food security) e à segurança sanitária (food safety)" (Lucchese, 2003, p.253). Entre as críticas endereçadas ao código, encontram-se a polêmica regulação dos alimentos geneticamente modificados e a sub-representação crônica dos países em via de desenvolvimento em suas negociações (Mbengue; Thomas, 2004, p.244).

As controvérsias em torno dos padrões do Codex envolvem consumidores, indústria, cientistas, médicos especialistas e governos, em todo o mundo, eis que a segurança dos alimentos é uma construção social, que depende de numerosas correlações de força entre os diversos atores, com destaque para as relações entre as diferentes formas de conhecimento científico e os atores que desenvolvem esse conhecimento (Ramsingh, 2014, p.215). Assim, o Codex reflete as pressões econômicas e políticas em prol da introdução crescente de novas tecnologias na produção de alimentos, podendo prescindir da investigação científica exaustiva, que avalie e delimite os riscos sobre a saúde humana e ambiental, consagrando a lógica segundo a qual é preciso regulamentar, mas não contrariar o ritmo das tecnologias que aumentam a produtividade e o lucro (Figueiredo; Miranda, 2011, p.2255).

Nesse sentido, o Codex Alimentarius é uma das vertentes do vasto processo internacional de regulamentação sanitária que melhor expressa os efeitos da globalização, pois "deixa pouco espaço para a busca e implantação de alternativas, arranjos e mediações domésticas às exigências do mercado internacional", capturando as "frágeis instituições nacionais dos países em desenvolvimento - o parlamento, as instâncias negociadoras da área das relações exteriores, as agências regulatórias e assim por diante" (Lucchese, 2003, p.254). Esse fenômeno é ainda mais inquietante quando se considera que a tomada de decisão, no âmbito 
intergovernamental, "muda o contexto" das relações entre os setores público e privado nacionais, pois a necessidade de construir coalizões encoraja o governo a cooperar com a indústria, que, por sua vez, a fim de aumentar as chances de ter seus interesses contemplados, tende a assumir os custos desta cooperação, resultando em relações "por vezes muito estreitas entre empresas e governos" (Lassalle-de Sallins, 2008, p.406). Não é difícil entender que o determinismo econômico reine no plano das organizações multilaterais da área econômica e financeira, mas é surpreendente que ele predomine também em organizações internacionais da área social, pois "a Comissão do Codex tem suas raízes na FAO e na OMS, cujas missões são muito mais abrangentes e importantes sob o ponto de vista humano. Por isso, deveria buscar um caminho de maior autonomia em relação aos interesses econômicos imediatos, dos países-membros e de suas empresas" (Lucchese, 2003, p.554).

\section{Concluindo}

Há um processo produtivo alimentar, crescentemente globalizado e dominante, com incontáveis e complexas ramificações e implicações sobre a sustentabilidade, a cultura, a sociedade, a economia e, mais importante, sobre a saúde e o bem-estar humano. "O superconsumo de alimentos não saudáveis se dá às custas da resiliência do planeta" (The Lancet, 2016).

No entanto, há várias dimensões que a relação entre alimentação e sustentabilidade assume. A alimentação está relacionada a praticamente todos Objetivos do Desenvolvimento Sustentável, que dependem, portanto, de uma mudança radical em nosso sistema alimentar para serem atingidos.

É muito comum a visão que resume tal relação aos aspectos ambientais/ ecológicos da alimentação, importantíssimos, porém não únicos. É necessário levar em conta os aspectos culturais, sociais e econômicos da sustentabilidade do sistema alimentar.

\section{Referências}

CAROLAN, M. The sociology of food and agriculture. London; New York: Routledge, 2012.

CARSON, R. Silent Spring. Greenwich, Conn., USA: Fawcett Publications, 1962.

CASSOL, A.; SCHNEIDER, S. Produção e consumo de alimentos: novas redes e atores. Lua Nova: Revista de Cultura e Política, São Paulo, n.95, maio-ago 2015. Disponível em: <http://dx.doi.org/10.1590/0102-6445143-177/95>. Acesso em: 10 abril 2016.

CONTE, I. I.; BOFF, L. A. As crises mundiais e a produção de alimentos no Brasil. Acta Scientiarum: Human and Social Sciences, v.35, n.1, p.49-59, 2013.

CULOT, H. Soft law et droit de l'OMC. Revue Internationale de Droit Économique, v. XIX, n.3, p.25l-89, 2005. 
CROVETTO, M. et al. Disponibilidad de productos alimentarios listos para el consumo em los hogares de Chile y su impacto sobre la calidad de la dieta (2006-2007). Revista Médica de Chile, v.142, p.850-8, 2014.

FAO - ORGANIZAÇÃO DAS NAÇÕES UNIDAS PARA A ALIMENTAÇÃO E A AGRICULTURA. Sustainable diets and biodiversity: directions and solutions for policy, research and action. Biodiversity and sustainable diets united against hunger. Rome: Food and Agricultural Organization, 2010.

FIGUEIREDO, A.; MIRANDA, M. Análise de Risco aplicada aos alimentos no Brasil: perspectivas e desafios. Ciênc. Saúde Coletiva, v.16, n.4, p.2251-62, Apr. 2011.

HALABI, S. The Codex Alimentarius Commission, Corporate Influence, and International Trade. American Journal of Law and Medicine, v.41, n.2, May 2015.

KATHOUNIAN, C. A. A reconstrução ecológica da agricultura. Agroecológica, Botucatu, 2001. 345p.

LAPPÉ, F. M. Diet for a small planet. Revised edition. New York: Ballantine Books, 1976. $411 \mathrm{p}$.

LASSALLE-DE SALINS, M. Les délégués des États dans les processus décisionnels des organisations intergouvernementales: la défense d'une position nationale au sein du Codex Alimentarius. Revue Française d'Administration Publique, v.2, n.126, p.387406, 2008.

Réduire l'incertitude sur le résultat des négociations intergouvernementales: les apports d'une lecture organisationnelle. Le cas du Codex Alimentarius. Négociations, v.1, n.11, p.143-58, 2009.

LUCCHESE, G. A internacionalização da regulamentação sanitária. Ciênc. Saúde Coletiva, v.8, n.2, p.537-55, 2003.

MALUF, R. S. et al. Nutrition-sensitive agriculture and the promotion of food and nutrition sovereignty and security in Brazil. Ciênc. Saúde Coletiva, v.20, n.8, p.2303$12,2015$.

MARTINS, A. P. B. et al. Participação crescente de produtos ultraprocessados na dieta brasileira (1987-2009). Revista de Saúde Pública, v.47, n.4, p.656-65, 2013.

MARTINELLI JUNIOR, O. O quadro regulatório dos mercados internacionais de alimentos: uma análise de seus principais componentes e determinantes. Econ. Soc., v.22, n.2, p.521-45, Aug. 2013.

MBENGUE, M.; THOMAS, U. Le Codex Alimentarius, le protocole de Cartagena et l'OMC: une relation triangulaire en émergence? Revue Européenne des Sciences Sociales, v.XLII, n.130, p.229-48, 2004.

MCSWANE, D. Seguridad de los alimentos. In: FRUMKIM, H. (Ed.) Salud Ambiental de lo global a lo Local. Organización Panamericana de la Salud. Washington D.C., 2010. p.633-82.

MONTEIRO, C. A. et al. Ultra-processed products are becoming dominant in the global food system. Obesity Reviews, v.14, n.2, p.21-8, 2013.

MONTEIRO, C. A. et al. Dietary guidelines to nourish humanity and the planet in the twenty-first century. A blueprint from Brazil. Public Health Nutrition, v.18, n.13, p.2311-22, 2015 . 
MONTEIRO, C. A. et al. The star shines bright. Journal of the World Public Health Nutrition Association, v.7. n.1-3, p.28-38, 2016.

MONTEIRO, C. A.; CANNON, G. The Impact of Transnational "Big Food" Companies on the South: A View from Brazil. PLoS Med, v.9, n.7, 2012.

MORIN, E. A Via para ofuturo da humanidade. Rio de Janeiro: Bertrand Brasil, 2013. 392p.

MOUBARAC, J. C. et al. International differences in cost and consumption of ready-to-consume food and drink products: United Kingdom and Brazil, 2008-2009. Global Public Health, v.8, n.7, p.845e856, 2013.

MOUBARAC, J. C. et al. Processed and ultra-processed food products: Consumption trends in Canada from 1938 to 2011. Canadian Journal of Dietetic Practice and Research, v.75, n.1, p.15 e 21, 2014.

NAVOLAR, T. S.; RIGON, S. A.; PHILIPPI, J. M. S. Diálogo entre agroecologia e promoção da saúde. Revista Brasileira de Promoção da Saúde, v.23, n.1, p.69-79, 2010.

OMS; FAO. Codex Members and Observers. Disponível em <http://www.fao.org/fao-who-codexalimentarius/members-observers/en/>. Acesso em: 8 jun. 2016.

PAHO/WHO. Ultra-processed products in Latin America: trends, impact on obesity, policy implications. Washington, DC: Pan American Health Organization / World Health Organization, 2015. 68p. [in press].

POLLAN, M. Em defesa da comida: um manifesto. Rio de Janeiro: Intrínseca, 2008. $275 p$.

POPKIN, B. M. Global nutrition dynamics: the world is shifting rapidly toward a diet linked with noncommunicable diseases. American Journal of Clinical Nutrition, v.84, n.2, p.289-98, 2006.

. Nutrition, agriculture and the global food system in low and middle income countries. Food Policy, v.47, p.91-6, 2014.

RAMSINGH B. The emergence of international food safety standards and guidelines: understanding the current landscape through a historical approach. Perspectives in Public Health, n.134, p.206-15, July 2014.

ROBSON, M.; HAMILTON. G. C. Control de Plagas y Pesticidas. In: FRUMKIM, H. (Ed.) Salud Ambiental de lo global a lo Local. Washington D.C.: Organización Panamericana de la Salud, 2010. p.593-632.

SCHNEIDER, S. Situando o desenvolvimento rural no Brasil: o contexto e as questões em debate. Revista de Economia Política, v.30, n.3 (119), p.511-31, jul.-set. 2010.

THE LANCET. Comment. Acting in the Anthopocene - The EAT Lancet Commission, n.387. 2016

WHO - WORLD HEALTH ORGANIZATION. Diet, nutrition and the prevention of chronic diseases: report of a joint. WHO/FAO Expert consultation. Geneva: World Health Organization, 2003.

WHO - WORLD HEALTH ORGANIZATION; UN-HABITAT. Global Report on Urban Health; equitable, healthier cities for sustainable development. Executive Summary. Centre for Health Development, Kobe; UN-Habitat, Nairobi, 2016 
WORLD BANK 2010. Urban Development Series. Waste Generation. Disponível em: <http://siteresources.worldbank.org/INTURBANDEVELOPMENT/Resources/336 387-1334852610766/Chap3.pdf>. Acesso em: 2 jun. 2013.

RESUMO - Em todo amplo, diverso e complexo trajeto do alimento, do solo ao prato, há inúmeras interfaces com a in/sustentabilidade. O tema da alimentação e sua relação com a sustentabilidade planetária é antigo e, ao mesmo tempo, muito atual, portanto de fronteira, no sentido temporal, de vanguarda. Ao exigir olhares, investigações e soluções multidisciplinares é, também, um tema de fronteira de conhecimentos, por conta de suas múltiplas interfaces. Este ensaio, de autoria interdisciplinar, tem como objetivo pincelar um panorama e apresentar uma reflexão sobre as complexas e intrincadas questões que envolvem a alimentação, sob uma perspectiva da sustentabilidade global. Aborda, inicialmente, a produção de alimentos no campo, com crescente quimificação e ampliação de áreas para produção agropecuária. Em seguida, tece relações entre o ambiente alimentar urbano e a crescente incidência de obesidade e sobrepeso, enquanto ainda persistem populações mal alimentadas. Aborda, a seguir, o novo paradigma sobre Alimentação Saudável e finaliza por discutir o Codex Alimentarius, o corpus juris mais importante no âmbito do comércio mundial de produtos alimentares. Conclui por reafirmar que há várias dimensões da relação entre alimentação e sustentabilidade, além da ecológica.

PALAVRAS-CHAVE: Sistema alimentar, Sustentabilidade, Regulação de alimentos, Saúde global.

ABSTRACT - In the vast, diverse and complex route of food from the soil to our plate, there are many interfaces with un/sustainability. The subject of food and its relation to planetary sustainability is old, and, at the same time, very new, and therefore can be considered a frontier field of study. Because it requires different sciences and multidisciplinary solutions, it can also be considered at the frontier of knowledge. The aim of this essay, of interdisciplinary authorship, is to paint an overview and offer a reflection on the complex issues involving the food system from the perspective of global sustainability. Initially, it presents a panorama of food production in the countryside, with growing use of chemicals products and the increase of livestock farming. Secondly, it shows relations between the urban food environment, obesity and incidence of noncommunicable diseases, while millions of people still go hungry. It then presents the new Health Food paradigm and discusses the Codex Alimentarius, the most important corpus juris for international food trade. And it ends by stating that there are various dimensions to relationship between food and sustainability besides the ecological one.

KEYWORDS: Food system, Sustainability, Food regulation, Global health.

Helena Ribeiro é geógrafa. Departamento de Saúde Ambiental, Programa de Pós-Gra-duação em Saúde Global e Sustentabilidade. Faculdade de Saúde Pública da Universidade de São Paulo. @-lena@usp.br

Patrícia Constante Jaime é Nutricionista. Departamento de Nutrição, Programa de Pós-Graduação em Saúde Global e Sustentabilidade. Faculdade de Saúde Pública da Universidade de São Paulo. @-constant@usp.br 
Deisy Ventura é advogada. Departamento de Saúde Ambiental. Programa de Pós-Gra-duação em Saúde Global e Sustentabilidade. Faculdade de Saúde Pública e Instituto de Relações Internacionais da Universidade de São Paulo. @-deisy.ventura@usp.br

Recebido em 31.8.2016 e aceito em 24.10.2016.

${ }^{\text {I }}$ Faculdade de Saúde Pública, Universidade de São Paulo, São Paulo / São Paulo, Brasil. 